\title{
THE EFFECTS OF ISOFLURANE ON ARTERIAL PRESSURE, PULSE RATE, AUTONOMIC NERVOUS ACTIVITY, AND BAROSTATIC REFLEXES
}

\author{
Per Skovsted and Sanguan Sapthavichaikul $\dagger$
}

\section{INTRODUCTION}

IsofLURANE (Forane ${ }^{\mathbb{S}}$ ) has been shown to depress myocardial contractility in the isolated papillary muscle of cats. ${ }^{1}$

The cardiovascular effects of isoflurane in $\operatorname{man}^{2}$ are somewhat perplexing. Isoflurane resembles agents like halothane ${ }^{3}$ and enfluranc ${ }^{4}$ known to depress sympathetic nervous activity because it causes both hypotension and vasodilatation. On the other hand, it resembles agents which stimulate sympathetic nervous activity, like diethyl ether, ${ }^{5,6}$ in the sense that it maintains cardiac output and causes tachycardia.

This study has been undertaken to evaluate to what extent changes in autonomic nervous activity might explain the cardiovascular effects of isoflurane.

\section{MethoD}

Twenty-seven cats were used. Details of the experimental technique have been described elsewhere. ${ }^{3}$ Briefly, basal anaesthesia consisted of 50 per cent nitrous oxide in oxygen with intravenous decamethonium 2 to $4 \mathrm{mg}$ initially and repeated every half-hour throughout the experiment. Respiration was controlled by a ventilator and end-tidal carbon dioxide was measured by a capnograph and maintained constant by adjusting the ventilator. Arterial pressure was measured from a femoral artery catheter connected to a Statham P-23 transducer. Mean arterial pressure was obtained by electrical damping. Autonomic nervous activity was recorded from teased strands of either the central part of the left cervical sympathetic trunk divided just below the superior cervical ganglion, or from teased strands of the proximal part of a sectioned left vagus nerve. Only sympathetic fibers showing a marked decrease in activity in response to the increase in arterial pressure produced by an intravenous injection of epinephrine were studied further, but vagal fibers were studied whether or not they showed an increase in activity under these circumstances. During the administration of

From Department of Anesthesiology, Hahnemann Medical College and Hospital of Philadelphia, PA 19102.

Portions of this work were presented at the Annual Meeting of the American Society of Anesthesiologists in Washington, D.C., October 1974.

Supported in part by NIH Grant HL 15392-03.

-Assistant Professor of Anaesthesiology. Present address: Department of Anaesthesiology, Rigshospitalet (University Hospital), Blegdamsvej 9, 2100 Copenhagen, Denmark.

†Senior Instructor of Anaesthesiology. 


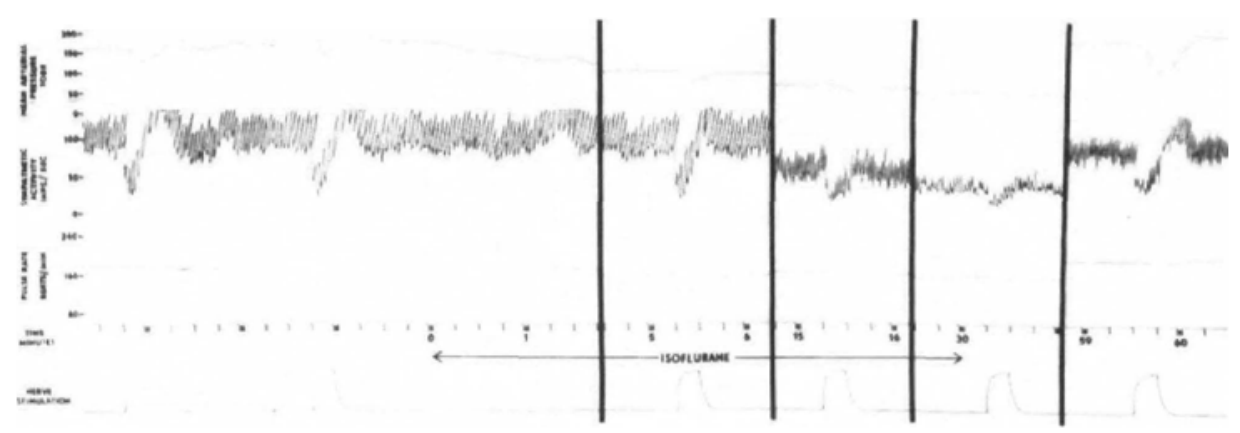

Ficune 1. Effects of increasing depths of isoflurane anaesthesia on arterial pressure, sympathetic nervous activity, and heart rate and on the responses of these to a 15-second period of aortic depressor nerve stimulation in a baroreceptor-denervated cat. Inspired isoflurane concentration was 1.2 per cent during the time interval $0-15 \mathrm{~min}$ and 2 per cent during the time interval $15-30 \mathrm{~min}$.

A progressive decline in arterial pressure, sympathetic nervous activity, and pulse rate is noted with increasing depth of anaesthesia. It is clearly demonstrated that during isoflurane administration aortic depressor nerve stimulation causes a depression in sympathetic activity even when it is already markedly depressed but without causing any conspicuous changes in arterial pressure.

isoflurane, barostatic reflexes were tested by electrical stimulation of the aortic depressor nerve. Stimulus characteristics and voltage used for the 15-second train-length stimulations also have been described previously. ${ }^{3}$ Body temperature was kept between $36^{\circ}$ and $38^{\circ} \mathrm{C}$ by a heating pad. Arterial blood samples were analyzed periodically in an I.L. assembly unit for $\mathrm{Po}_{2}, \mathrm{Pco}_{2}$, and $\mathrm{pH}$. Metabolic acidosis, if present, was corrected by infusion of sodium bicarbonate.

Isoflurane was administered from a copper kettle. The inspired concentration was 1.2 per cent for the first 15 minutes followed by 2 per cent for an additional 15 minutes. At the 5th, 15th and 30th minutes of exposure, end-tidal isoflurane concentrations were measured by gas chromatography.

The following parameters were displayed continuously on a Grass multichannel recorder: arterial pressure, mean arterial pressure, end-tidal $\mathrm{CO}_{2}$, nerve activity, and pulse rate. Measurements requiring calculation were obtained before the administration of isoflurane (control) and then at the 5 th, 15th, and 30 th minute. Final measurements were made 30 minutes after isoflurane had been discontinued. In seven animals, a good response to baroreceptor nerve stimulation was obtained. The sensitivity of barostatic reflexes has been expressed as the percentage depres- 
sion of mean arterial pressure and pulse rate. The sympathetic response was calculated somewhat differently, namely the percentage of impulses deleted from the activity-time curve during stimulation. We find this value more meaningful than the maximal response, since sympathetic activity often failed to remain inhibited through the entire period of stimulation (Figure 1). All initial and final values recorded for barostatic responses are the mean of two measurements.

\section{Experimental Protocol}

Ten "normal" animals were studied. A "normal" animal is defined as one in which only the sympathetic trunk was sectioned.

Ten animals were studied in a baroreceptor-denervated state. In five of these animals a surgical section of all barostatic nerves was carried out. The remaining five were animals previously exposed to isoflurane in a "normal" state and subsequently denervated by section of the vagal and aortic depressor nerves and carotid artery ligation below the carotid bulbs. In all cases, denervation led to an increase in arterial pressure, sympathetic nervous activity and pulse rate. Forty-five to fifty minutes were allowed for all parameters to stabilize before the animals were exposed again to isoflurane.

Six "vagal" animals were studied. A vagal animal is defined as one in which only the left vagus nerve was sectioned.

Six animals were studied after mid-collicular decerebration. The study was carried out as in "normal" animals, except that the lungs were ventilated with oxygen instead of nitrous oxide and oxygen. All statistical analyses were performed using t-tests. A p-value of less than 0.05 was considered statistically significant.

\section{Results}

The results are listed in Tables I, II, III and on Figure 2. All values are means and standard errors of the results obtained from each group of animals. Significant changes from initial values are indicated by a p-value.

\section{Normal animals}

After five minutes with an end-expired isoflurane concentration of $0.69 \pm 0.03$ per cent, sympathetic activity had declined from $119 \pm 17$ to $86 \pm 15 \mathrm{impulses} / \mathrm{sec}$ $(\mathrm{p}<0.005)$. After 15 minutes the end-expired isoflurane concentration was $0.98 \pm 0.04$ per cent and the level of sympathetic activity was not essentially different from the five-minute value. After 30 minutes with an end-expired isoflurane concentration of $1.82 \pm 0.06$ per cent, sympathetic activity had declined further to $34 \pm 7$ impulses $/ \mathrm{sec}(p<0.001)$. In general, a modest decline in sympathetic activity was seen with isoflurane concentrations up to 1 per cent, followed by a uniformly marked depression when the isoflurane concentration was increased to 1.8 per cent at which point sympathetic tone averaged only 30 per cent of the initial level.

After five minutes the response of mean arterial blood pressure to isoflurane at concentrations up to 1 per cent was a modest decline from initial values of 


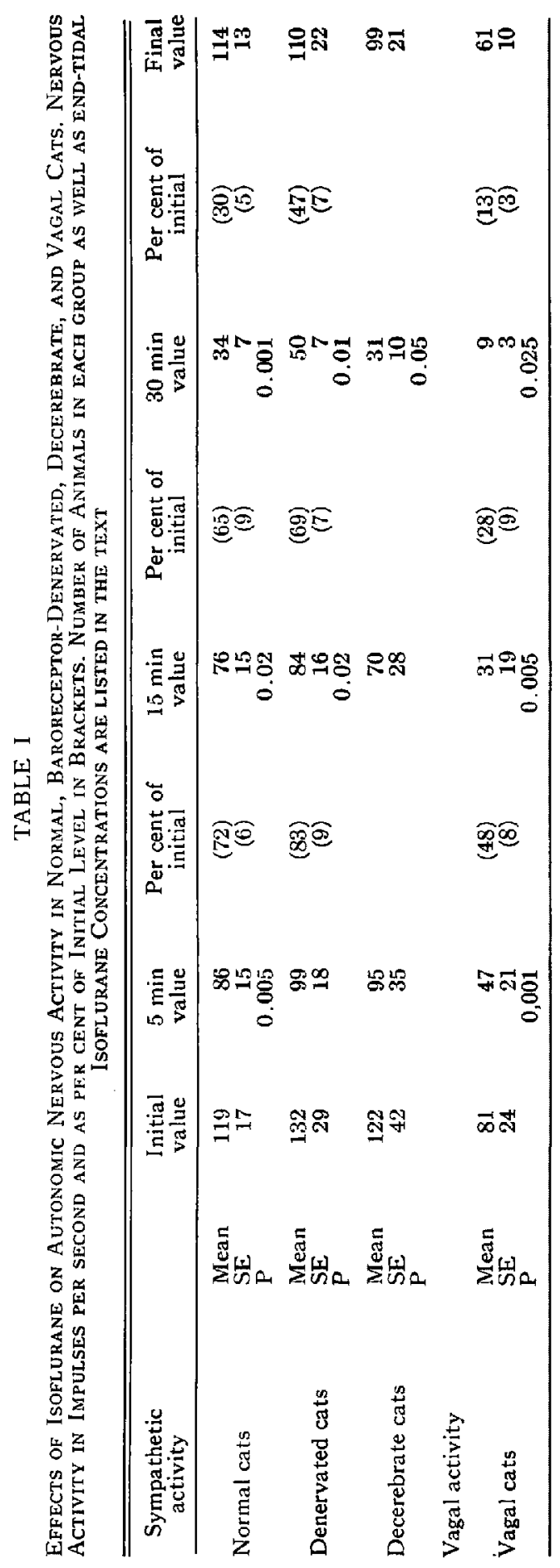




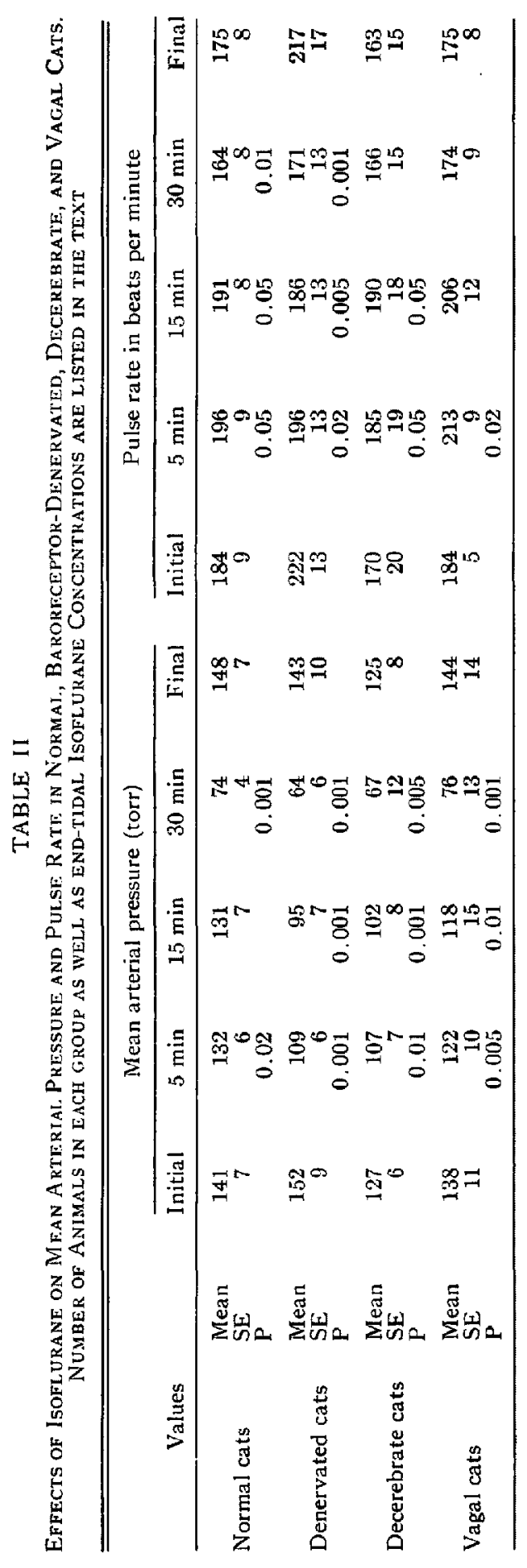


TABLE III

Effects of Isoflurane on the Responses of Sympathetic Nervous Activity, Mean Arterial Blood Pressure, and Heart Rate to Aortic Depressor Nerve Stmulation*

\begin{tabular}{lrrrrr}
\hline \hline & $\begin{array}{c}\text { Initial } \\
\text { control }\end{array}$ & $\begin{array}{c}5 \mathrm{~min} \\
\text { value }\end{array}$ & $\begin{array}{c}15 \mathrm{~min} \\
\text { value }\end{array}$ & $\begin{array}{c}30 \mathrm{~min} \\
\text { value }\end{array}$ & $\begin{array}{r}\text { Final } \\
\text { value }\end{array}$ \\
\hline Sympathetic Activity & 30.2 & 34.3 & 25.7 & 23.5 & 35.2 \\
Response & \pm 6.0 & \pm 4.7 & \pm 3.1 & \pm 5.8 & \pm 7.2 \\
Mean Arterial Pressure & 17.2 & 16.9 & 13.5 & 8.0 & 11.3 \\
Response & \pm 4.7 & \pm 5.0 & \pm 5.5 & \pm 3.3 & \pm 2.3 \\
Heart Rate & 7.4 & 6.5 & 6.9 & 4.0 & 4.7 \\
Response & \pm 3.3 & \pm 2.9 & \pm 3.6 & \pm 2.6 & \pm 2.0 \\
\hline
\end{tabular}

*All values are means from seven animals expressed as percentage reductions (see text) \pm standard errors.

$141 \pm 7$ to $132 \pm 6$ torr $(p<0.02$ ). After 30 minutes, however, a marked reduction in mean arterial blood pressure was observed ( $74 \pm 4$ torr).

The pulse rate increased after five minutes and 15 minutes of isoflurane $(p<0.05)$. When the end-expiratory isoflurane concentration was increased to 1.8 per cent the pulse rate fell to $164 \pm 8$ beats $/ \mathrm{min}(\mathrm{p}<0.01)$ at the 30 -minute measurement.

\section{Denervated Animals}

Sympathetic activity and mean arterial blood pressure declined with increasing isoflurane concentrations as in the normal animals; but, unlike the normal animals, denervated animals never showed any increase in pulse rate. On the contrary, a progressive decline in pulse rate was always observed with increasing concentrations of isoflurane. The end-expired isoflurane concentrations were $0.76 \pm 0.04$ per cent after five minutes, $1.00 \pm 0.06$ per cent after 15 minutes, and $1.85 \pm 0.02$ per cent after 30 minutes.

\section{Vagal Animals}

The response of arterial pressure and pulse rate of vagal animals exposed to isoflurane was similar to that found in "normal" animals. The response in vagal activity showed a progressive decline with increasing isoflurane concentrations. From an initial $81 \pm 24 \mathrm{impulses} / \mathrm{sec}$ to $47 \pm 21 \mathrm{impulses} / \mathrm{sec}$ after five minutes $(\mathrm{p}<0.001)$, to $31 \pm 19$ impulses/sec after 15 minutes $(p<0.005)$ and finally to $9 \pm 3$ impulses/sec after 30 minutes $(p<0.025)$ of isollurane exposure.

Using the percentage of initial level of nervous activity, it is seen that at all isoflurane concentrations sympathetic tone was significantly less depressed than vagal tone. At five minutes sympathetic activity was $72 \pm 6$ per cent of the initial level as against $48 \pm 8$ per cent for vagal activity $(p<0.05)$. At 15 minutes the respective values were $65 \pm 9$ per cent and $28 \pm 9$ per cent $(p<0.02)$, and at 30 minutes $30 \pm 5$ per cent versus $13 \pm 4$ per cent $(p<0.05)$. The end-expired isoflurane concentrations were $0.71 \pm 0.02$ per cent after five minutes, $0.93 \pm 0.04$ per cent after 15 minutes, and $1.72 \pm 0.04$ per cent after 30 minutes. 

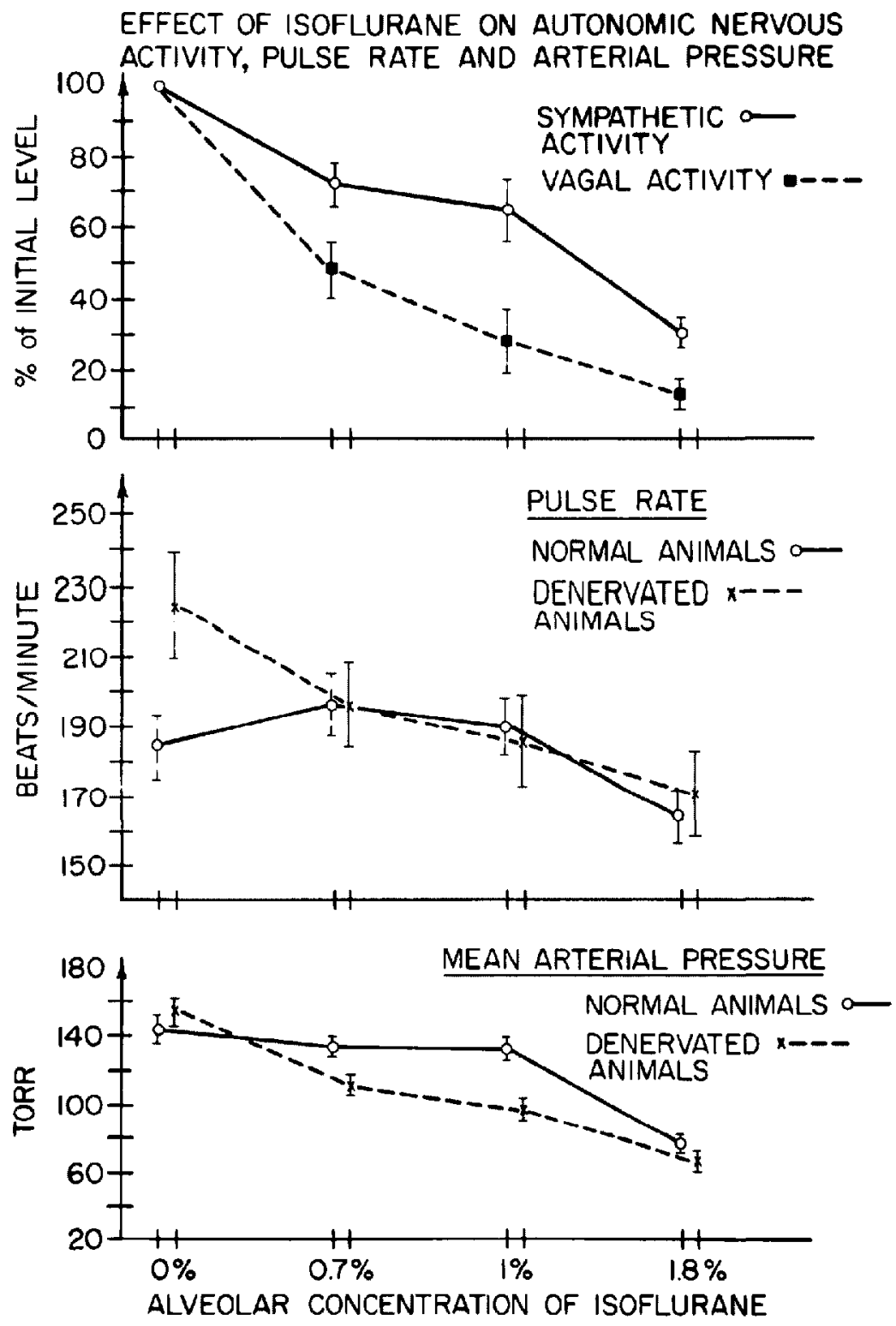

Ficune 2. Effects of isoflurane on sympathetic nervous activity, mean arterial pressure, and pulse rate in nomal, baroreceptor-denervated, and vagal cats. The essential findings from Tables I and II are jllustrated in graphic form. Upper part shows that isoflurane causes considerably more depression in vagal than in sympathetic tone. Middle part illustrates that isoflurane at increasing concentrations causes a progressive reduction in pulse rate in the denervated cats, whereas in normal cats at moderate isoflurane concentrations tachycardia is seen. Lower part: baroreceptor-denervated cats react to increasing isoflurane concentrations with progressive decline in arterial pressure whereas nomal cats show less decline in arterial pressure as long as tachycardia is present. 


\section{Decerebrate Animals}

The response to isoflurane in decerebrate animals was essentially the same as that found in intact animals.

\section{Barostatic Reflexes}

For the effect of isoflurane on the response to stimulation of the aortic depressor nerve in the seven animals in which a good initial response was obtained, see Table III.

Isoflurane did not alter the response in sympathetic activity or pulse rate. The response in mean arterial blood pressure was significantly reduced from 17.2 to 8 per cent $(p<0.01)$ but this occurred only at the high isoflurane concentration of 1.8 per cent.

\section{Discussion}

This study has three major objectives.

The first objective is to examine whether the depression of blood pressure that has been shown to occur with isoflurane anaesthesia ${ }^{2.7}$ is due solely to a cardiovascular depressant action of the agent or if depression of the sympathetic nervous system also plays a role, as had been found lor enflurane, the isomer of isoflurane. ${ }^{4}$

The second objective is to examine whether the tachycardia occurring during isoflurane anaesthesia is due to a sympathomimetic action of the agent, as has been suggested by two other groups of investigators. Stevens, et al ${ }^{2}{ }^{2}$ studying unpremedicated volunteers, hypothesized that a direct $\beta$-receptor stimulating effect of isoflurane might be involved. In a study of patients undergoing operations under isoflurane anaesthesia, Graves, et al. ${ }^{\top}$ deduced, from maintained cardiac output and increased total peripheral resistance and pulse rate, that the action of isoflurance on the autonomic nervous system resembles that of diethyl ether, which has a combined sympathomimetic and vagolytic action. However, an action on the parasympathetic part of the autonomic nervous system alone could have been responsible for the tachycardia, a possible explanation ignored by these authors.

The third objective is to examine whether changes in barostatic reflexes contribute to, or are responsible for, the cardiovascular changes occurring during isoflurane anaesthesia.

Only cervical sympathetic and vagal nerve fibers were studied and we have no evidence that they are absolutely representative of the action of isoflurane on the autonomic nervous system in general. From the work of Millar and Biscoe ${ }^{8}$ it appears likely that the effect of anaesthetics on the sympathetic nervous system is general and that cervical sympathetic fibers are representative. This assumption has recently been substantiated by Roizen, et $a l^{9}$ who, with a new sensitive radiometric assay of catecholamines, confirmed our previous finding in cats ${ }^{3}$ that halothane causes a reduction in sympathetic nervous activity.

It is technically exceedingly difficult to isolate the vagal fibers going to the heart in the cat. One might argue that our use of reactive fibers from a vagus nerve is not representative of the parasympathetic nervous system response to anaesthetic 
agents, since this assumes that the response of the system to anaesthetic agents is uniform. There is indirect evidence that indeed this is the case, as shown by Christensen and Skovsted. ${ }^{10}$ They found that the $\mathrm{pH}$ of gastric content increased with anaesthetic agents which reduce vagal tone and remained unchanged with agents increasing vagal tone, the vagus being one of the prime regulators of acid secretion in the stomach. Thus, we believe that the parasympathetic nervous system, like the sympathetic nervous system, reacts to anaesthetic agents in a uniform manner and that the isoflurane-induced depression of vagal tone which we observed, is true also for vagal fibers innervating heart.

In agreement with the findings of Stevens, et al. ${ }^{2}$ a substantial depression of blood pressure, increasing with anaesthetic depth, was found during isoflurane anaesthesia. Sympathetic activity was similarly affected, suggesting that at least part of the cardiovascular depression caused by isoflurane is the result of reduced sympathetic tone. The observation that baroreceptor nerve stimulation during isoflurane anaesthesia not only reduced sympathetic tone but also reduced arterial pressure supports this theory. Since decerebrate animals reacted the same way as normal animals, in the absence of basal anaesthesia, we can assume that neither our basal anaesthetic nor the effect of isoflurane on autonomic centers in the hypothalamus played any major role in the autonomic nervous response obtained. In accordance with our previous method of interpretation based on the findings of Alexander ${ }^{11}$ we must assume that isoflurane acts on the vasomotor center in the medulla oblongata, depressing the pressor neurons of this center. When during isoflurane anaesthcsia cardiac output is maintained because tachycardia compensates for decreased stroke volume, in spite of depressed sympathetic tone, the question centers around the cause of this tachycardia. Since our denervated animals showed a progressive decline in pulse rate it might be assumed that, even if isoflurane acted as a direct $\beta$-receptor stimulant as suggested by Stevens et al.,2 this effect alone cannot compensate for the central sympathetic depression and the possible negative chronotropic effect of the agent. Our results showing central depression of vagal tone significantly greater than the depression of sympathetic tone seem to indicate that depression of the parasympathetic nervous system is at least partially responsible for the tachycardia seen during isoflurane anaesthesia and that this depression occurs centrally. A concomitant peripheral atropine-like action cannot be ruled out; such an action can only be verified or disproven by additional studies. On the basis of this study, it appears most likely that no single pharmacological factor is responsible for the changes in pulse rate seen during isoflurane anaesthesia. In all probability we are dealing with multiple interacting factors of which the observed greater depression of parasympathetic as compared to sympathetic tone is only one. The finding of bradycardia with high anaesthetic concentrations in "denervated" animals and even "normal" animals is presumably due to a direct negative chronotropic effect of isoflurane which becomes evident when autonomic nervous system activity is either severely depressed or abolished. Krishna, et al. ${ }^{12}$ have recently shown that anaesthetic agents might directly affect the intrinsic frequency of contractions of atrial preparations, independent of the autonomic nervous system and its humoral transmitters.

Our study clearly shows that sympathetic response to baroreceptor nerve stimu- 
lation is present at high isoflurane concentrations, but the response of arterial pressure to changes in sympathetic tone is depressed. Similar findings have been reported during halothane anaesthesia. ${ }^{3}$ This is believed to be due to decreased sensitivity of vascular smooth muscle to sympathetic impulses.

Our finding of active barostatic reflexes during isoflurane anaesthesia leaves open the question of why, if barostatic reflexes are active, sympathetic nervous activity does not increase in response to hypotension caused by direct cardiovascular depression in the "normal" animals. Sensitization of peripheral baroreceptors caused by anaesthetics as shown by Biscoe and Millar ${ }^{13}$ may play a part. Most likely we are dealing with a non-linear system where depression in sympathetic nervous activity due to increased arterial pressure or baroreceptor nerve stimulation in no way assures an increased sympathetic tone in response to lowered arterial pressure.

Based on the findings of this study isoflurane appears to resemble halothane in its cardiovascular actions, as it reduces sympathetic nervous activity by acting on the pressor neurons in the vasomotor center and decreases the sensitivity of vascular smooth muscle to sympathetic impulses. It differs from halothane in that it causes tachycardia, which we believe is due in part to a greater depression of the parasympathetic than of the sympathetic nervous system.

The clinical implications of these findings are that when vagal blockade is produced by sufficient doses of atropine given before anaesthesia isoflurane would be expected to resemble halothane in its cardiovascular action, producing both hypotension and decreased cardiac output, since tachycardia compensating for the diminished stroke volume would be either non-existent or greatly diminished compared to events in unpremedicated man. On the other hand, it does appear logical to counteract any severe depression in arterial pressure caused by isoflurane by administration of a vasopressor, because autonomic nervous activity is so depressed that any baroreceptor reflex response to increased pressure can hardly be expected to cause any significant change in the autonomic nervous control of cardiac output, which would sacrifice flow for pressure. On the contrary one would expect the peripheral vasoconstriction produced by phenylephrine or methoxamine to cause increased venous return to the heart, thereby increasing cardiac output.

\section{SUMMARY}

The effects of isoflurane on arterial pressure, preganglionic sympathetic activity, vagal nervous activity, pulse rate, and barostatic reflexes were studied in cats. When the end-expired isoflurane concentration did not exceed 1.0 per cent tachycardia was observed which was abolished when the anaesthetic concentration was increased to 1.8 per cent. Arterial pressure, sympathetic nervous activity and vagal activity declined progressively with increasing depth of anaesthesia, but the reduction of vagal activity at all levels of anaesthesia always significantly exceeded the reduction of sympathetic activity. Barostatic reflexes, measured as the reduction in arterial pressure, pulse rate and sympathetic nervous activity when an aortic depressor nerve was stimulated electrically, were found to be 
normal, except that the response of arterial pressure was depressed at high isoflurane concentration.

The authors therefore conclude that isoflurane depresses the cardiovascular system not only by direct action, but also by depression of the sympathetic nervous system. The tachycardia seen during isoflurane anaesthesia can be explained in part by a greater depression of parasympathetic than of sympathetic tone. The fact that barostatic reflexes were active during isoflurane anaesthesia while the reflex response of arterial pressure was diminished leads the authors to believe that isoflurane depresses the response of vascular smooth muscle to sympathetic impulses.

\section{ACKNOWLEDGMENTS}

The authors thank Henry L. Price, M.D., Professor, Chairman and Research Director of our department for help and advice during the study, Mr. Vitcha, Airco Inc., for providing isoflurane for the study, Mr. Leo Davidson for his able technical assistance, and Jonathan Korshin, M.D., for help in preparation of the manuscript.

\section{REFERENCES}

1. Kenmuto, O., Hashimoto, $Y$., \& Shimosato, S. Inotropic effects of isoflurane on mechanics of contraction in isolated cat papillary muscles from normal and failing hearts. Anesthesiology 39: 479 (1973).

2. Stevens, W.D., Crommell, T.H., Halsey, M.J., Eger, E.I., Shakespeane, T.F., \& BAHLMAx, S.H. The cardiovascular effects of a new inhalational anesthetic, forane, in human volunteers at constant arterial carbon dioxide tension. Anesthesiology 35: 8 (1971).

3. Skovsted, P., Price, M.L., \& Price, H.L. The effects of halothane on arterial pressure, preganglionic sympathetic activity and barostatic reflexes. Anesthesiology 31: 507 (1969).

4. Skovsted, P. \& Price, H.L. The effects of ethrane on arterial pressure, preganglionic sympathetic activity and barostatic reflexes. Anesthesiology 36: 257 (1972).

5. Skovsted, P. \& Price, H.L. Central sympathetic excitation caused by diethyl ether. Anesthesiology 32: 202 (1970).

6. Grecony, G.A., Eger, E.I., Ty Sinth, N. Cullen, B.F., \& Cullen, D.J. The cardiovascular effects of diethyl ether in man. Anesthesiology 34: 19 (1971).

7. Graves, L.C., McDenuotr, R.W., \& Bidwal, A. Cardiovascular effects of isoflurane in surgical patients. Anesthesiology 41:486 (1974).

8. Millan, R.A. \& Biscoe, T.J. Postganglionic sympathetic discharge and the effect of inhalational anesthetics. B.J. Anaesth. 38: 92 (1966).

9. Rolzen, M.F., Moss, J., HENry, D.P., \& Kopin, I.J. Effects of halothane on plasma catecholamines. Anesthesiology 41: 432 (1974).

10. Christensex, V.\& Skovsten, P. Effects of general anesthetics on the pH on gastric content in man during surgery. Acta Anaesth. Scandinav. 19: 49 (1975).

11. Alexaxder, R.S. Tonic and reflex function of medullary sympathetic cardiovascular centers. J. Neurophysiology 9: 205 (1946).

12. Krishiva, G., Truebloon, S., \& Pradise, R.R. The mechanism of the positive chronotropic action of diethyl ether on rat atria. Anesthesiology 42:312 (1975).

13. Biscos, T.J. \& MILLAR, R.A. The effect of halothane on carotid sinus baroreceptor activity. B.J. of Physiology 173: 24 (1964). 